\title{
Improving patient safety and quality of healthcare through prevention of healthcare associated infections
}

\author{
Mariela Deliverska, Andrey Kehayov
}

Faculty of Public Health, Medical University - Sofia

\begin{abstract}
The negative impact of healthcare associated infections form patient morbidity and mortality risks and also result prolongation in hospitalization of patients, which provides additional costs.

Healthcare associated infections, which are directly association with exposure to healthcare facilities or healthcare treatments or procedures, reduce the quality of life.

Exercising the right of every patient to put into force a complaint procedure in relation to negative results related to healthcare associated infection in the process of delivery of healthcare services could have a severe impact on the entire system. That is because the appearance of healthcare associated infection involves multiple parties, including the patient, the healthcare provider, the facility in which the patient is treated, the insurance company and health lawyers.
\end{abstract}

Keywords: Healthcare associated infections, prevention, patient safety, quality 
Healthcare associated infections are significant threat to patient safety in relation to providing healthcare services. Appearance of these types of diseases or pathologies is directly related to negative effects on the entire healthcare system due to resulting of poor quality level of services.

Healthcare associated infections could be defined as diseases or pathologies related to the presence of an infectious agent or its products. They are directly association with exposure to healthcare facilities or healthcare treatments or procedures.

The negative impact of healthcare associated infections form patient morbidity and mortality risks and also result prolongation in hospitalization of patients, which provides additional costs.

Healthcare associated infections always reduce quality of life, while in fact systematic factors account in the majority of cases related to poor patient safety. That is why cases of healthcare associated infections could be reduced and prevented, if competent authorities both on national and international level provide adequate measures.

In relation to the establishment actions, which are to complement national policies directly resulting public health improvement and prevention of human illness and diseases, in relation to article 152 of the Treaty establishing the European Community, in 2009 a Council recommendation ${ }^{1}$ has been introduced. This document has developed the base for establishment and development of national policies and programs on patient safety regarding healthcare associated infections (1).

The main focus has been put on the fact that the improvement of the patient safety process is directly related to the information and education of patients. Patients should be informed on the safety standards, the safety measures and the best practices. Patients should be aware of possible risks, available alternatives and safety measures when medical services are being provided to them. This information is directly related to the right to informed consent to treatment.

Exercising the right of every patient to put into force a complaint procedure in relation to negative results related to healthcare associated infection in the process of delivery of healthcare services could have a severe impact on the entire system. That is because the appearance of healthcare associated infection involves multiple parties, including the patient, the healthcare provider, the facility in which the patient is treated, the insurance company and health lawyers.

In order to achieve better results in limiting and preventing cases of healthcare associated infections, healthcare providers should be encouraged to actively report such cases. For that matter there need to be created a favorable reporting environment on national level. In relation to that the European union has introduced $^{2}$ systematic case definitions of healthcare associated infections, as well as instructions for reporting cases for various conditions (2).

\footnotetext{
${ }^{1}$ Council recommendation of 9 June 2009 on patient safety, including the prevention and control of healthcare associated infections, OJ C 151, 3.7.2009, p. 1-6.

${ }^{2}$ Commission Decision 2012/506/EU of 8 August 2012 includes in its annex general and specific systemic case definitions of $\mathrm{HAl}$, including reporting instructions for each of the conditions.
} 
The fact that delivery of healthcare services has been provided in non-hospital settings, including ambulatory surgical centers and private doctors' offices outlines the need of introducing an appropriate level of training and education of healthcare professionals on patient safety, including healthcare associated infections.

Organizations, providing healthcare services or support services at a local level, should also be involved in the process of providing information and education in efforts to eliminate and prevent healthcare associated infections.

Institutions and organizations providing education and training of medical and non-medical specialists, such as Medical universities and Colleges, should introduce into their curriculum special courses on healthcare associated infections in order to contribute to the improvement of patient safety and the quality of healthcare.

The introduction of hospital infection control programs also increases the effectiveness of improving patients safety in regard to healthcare associated infections and the overall level of quality of healthcare services.

According to special Eurobarometer survey related to Patient Safety and Quality of Care ${ }^{3}$, over $53 \%$ of all EU citizens express the opinion that patients could be harmed by hospital care in their country (3).

In Bulgaria, the prevention and control of healthcare associated infections has been introduces as an objective and since 2013, as part of the national legal framework has been introduced a medical standard on prevention and control of nosocomial infections ${ }^{4}$, approved with Ordinance No. 3 of May 8, 2013 (4). The standard takes into consideration the importance of surveillance of targeted infection types, which is directly related to the establishment of national reference database.

All properly relevant activities, related to prevention and control of healthcare associated infections, directly benefit the improvement of patient safety. These activities could essentially reduce the number of people affected by healthcare associated infections and improve the quality of healthcare services provided on national level.

\section{References}

1. European Commission, Report from the Commission to the Council, The Commission's Second report to the Council on the implementation of Council Recommendation on 2009/C 151/01 on patient safety, including the prevention and control of healthcare associated infections, Brussels, COM (2014) 371

\footnotetext{
${ }^{3}$ http://ec.europa.eu/health/patient_safety/eurobarometers/ebs_411_en.htm

${ }^{4}$ Ordinance No.3 of May 8, 2013 for approval of medical standard on prevention and control of nosocomial infections, State gazette 43 of May 14, 2013.
} 
2. Commission Implementing Decision of 8 August 2012 amending Decision 2002/253/EC laying down case definitions for reporting communicable diseases to the Community network under Decision No 2119/98/EC of the European Parliament and of the Council

3. European Commission, Eurobarometer Servey on Patient Safety and Quality of Care, 2014

4. Minister of Health of Republic of Bulgaria, Ordinance No.3 of May 8, 2013 for approval of medical standard on prevention and control of nosocomial infections, State Gazette 43 of May 14, 2013.

\section{Corresponding author:}

Assoc. prof. Mariela Deliverska, LLM, PhD, DMSc

DFaculty of Public Health

Medical University - Sofia

1527 Sofia, Bulgaria

8 Bialo more str. 